\title{
A Finite ANALOGUE OF THE RING OF ALGEBRAIC NUMBERS
}

\author{
JULIAN ROSEN
}

\begin{abstract}
We construct an analogue of the ring of algebraic numbers, living in a quotient of the product of all finite fields of prime order. We use this ring to deduce some results about linear recurrent sequences.
\end{abstract}

\section{INTRODUCTION}

A period is a complex number given as the integral of an algebraic function over a region defined by algebraic inequalities. The set of all periods is a countable subring of $\mathbb{C}$ containing $\overline{\mathbb{Q}}$ (see [8] for an overview of periods). Several recent works (e.g. [3, 4, 5, 10, 11, 13]) consider "finite" analogues of certain periods (finite multiple zeta values, finite multiple polylogarithms, etc.) living in the ring

$$
\mathcal{A}:=\frac{\prod_{p} \mathbb{Z} / p \mathbb{Z}}{\bigoplus_{p} \mathbb{Z} / p \mathbb{Z}}
$$

which was introduced by Konstsevich $([7], \S 2.2)$. An element of $\mathcal{A}$ is a primeindexed sequence $\left(a_{p}\right)_{p}$, with $a_{p} \in \mathbb{Z} / p \mathbb{Z}$, and two sequences are equal if they agree for all sufficiently large $p$. Every non-zero integer is invertible modulo $p$ for all sufficiently large $p$, so there is a diagonal embedding $\mathbb{Q} \hookrightarrow \mathcal{A}$.

1.1. Results. The purpose of this paper is to define a countable $\mathbb{Q}$-subalgebra $\mathcal{P}_{\mathcal{A}}^{0} \subset \mathcal{A}$ that is a finite analogue of $\overline{\mathbb{Q}} \subset \mathbb{C}$. This algebra is not the integral closure of $\mathbb{Q}$ inside $\mathcal{A}$, which has continuum cardinality.

Our first main result is three equivalent characterizations of $\mathcal{P}_{\mathcal{A}}^{0}$.

Theorem 1.1. The following subsets of $\mathcal{A}$ are equal.

(1) The set of elements $\left(a_{p} \bmod p\right)_{p}$, where $a_{0}, a_{1}, a_{2}, \ldots \in \mathbb{Q}$ is a recurrent sequence (that is, a sequence satisfying a linear recurrence relation with constant coefficients).

(2) The set of elements $\left(g\left(\phi_{p}\right) \bmod p\right)_{p}$, where $L / \mathbb{Q}$ is a finite Galois extension, $g: \operatorname{Gal}(L / \mathbb{Q}) \rightarrow L$ satisfies $g\left(\sigma \tau \sigma^{-1}\right)=\sigma(g(\tau))$, and $\phi_{p}$ is the $e^{1}$ Frobenius at $p$.

Date: November 13, 2019.

Key words and phrases. Finite periods, Frobenius automorphism, linear recurrence, congruence.

${ }^{1}$ This is independent of the representative of the Frobenius conjugacy class (see $\S 2$ ). 
(3) The set of $\mathbb{Q}$-linear combinations of matrix coefficients for the $\mathcal{A}$ valued Frobenius automorphism

$$
F_{\mathcal{A}}: L \otimes \mathcal{A} \rightarrow L \otimes \mathcal{A},
$$

defined by Definition 4.1, as $L$ ranges over all number fields.

The equivalence of (1) and (2) is Theorem 2.2, and the equivalence of (2) and (3) is Theorem 4.2.

Definition 1.2. We define $\mathcal{P}_{\mathcal{A}}^{0} \subset \mathcal{A}$ to be the set given by Theorem 1.1.

The Skolem-Mahler-Lech theorem says that if $\left(a_{n}\right)$ is a recurrent sequence, the set $\left\{n: a_{n}=0\right\}$ is has finite symmetric difference with a finite union of arithmetic progressions. As a consequence of Theorem 1.1, we obtain an analogue of Skolem-Mahler-Lech for the set of primes $\left\{p: a_{p} \equiv 0 \bmod p\right\}$. A set $P$ of primes is called Frobenian (cf. [12], §3.3) if there is a finite Galois extension $L / \mathbb{Q}$ and a union of conjugacy classes $C \subset \operatorname{Gal}(L / \mathbb{Q})$ such that $P$ has finite symmetric difference with the set of rational primes whose Frobenius conjugacy class is in $C$. The Chebotarev density theorem implies that the natrual density of a Frobenian set exists and is a rational number.

Corollary 1.3. A set $P$ of primes is Frobenian if and only if there exists a recurrent sequence $\left(a_{n}\right)$ such that

$$
P=\left\{p: a_{p} \equiv 0 \bmod p\right\} .
$$

Unlike the Skolem-Mahler-Lech Theorem, Corollary 1.3 is effective: given the recurrence relation satisfied by $\left(a_{n}\right)$ and a list of initial values, there is a finite algorithm to determine the number field $L$, union of conjugacy classes $C \subset \operatorname{Gal}(L / \mathbb{Q})$, and the finite exceptional set.

We also prove some results about polynomial equations satisfied by elements of $\mathcal{P}_{\mathcal{A}}^{0}$. The first of these results implies that $\mathcal{P}_{\mathcal{A}}^{0}$ is an integral extension of $\mathbb{Q}$.

Theorem 1.4. Suppose $\alpha \in \mathcal{P}_{\mathcal{A}}^{0}$. Then there exists a non-zero polynomial $f(x) \in \mathbb{Q}[x]$ such that $f(\alpha)=0$, and every such $f(x)$ has a rational root.

Remark 1.5. The Fibonacci sequence $F_{n}$ is known to satisfy the congruence $F_{p} \equiv\left(\frac{p}{5}\right) \bmod p$ for every prime $p$, where $\left(\frac{p}{5}\right)$ is a Legendre symbol. Thus $f\left(F_{p}\right) \equiv 0 \bmod p$ for $p \geq 7$, where $f(x)=x^{2}-1 \in \mathbb{Q}[x]$. Theorem 1.4 implies that every recurrent sequence satisfies an analogous identity for some $f$, which necessarily has a rational root.

We also prove a result about the density of the set of primes $p$ for which $f\left(a_{p}\right) \equiv 0 \bmod p$, when $\left(a_{p}\right) \in \mathcal{P}_{\mathcal{A}}^{0}$ and $f(x) \in \mathbb{Q}[x]$.

Theorem 1.6. For $f(x) \in \mathbb{Q}[x]$, we have

$$
\sup _{\left(a_{p}\right) \in \mathcal{P}_{\mathcal{A}}^{0}} \delta\left(\left\{p: f\left(a_{p}\right) \equiv 0 \bmod p\right\}\right)=\delta(\{p: f \text { has a root } \bmod p\})
$$


where $\delta$ denotes natural density. Moreover if $f(x)$ has no rational roots, then there is no element of $\mathcal{P}_{\mathcal{A}}^{0}$ realizing the supremum.

In $\S 4$ we explain the analogy between $\mathcal{P}_{\mathcal{A}}^{0} \subset \mathcal{A}$ and $\overline{\mathbb{Q}} \subset \mathbb{C}$, and the relationship with periods.

\section{Functions on a Galois group}

Let $L / \mathbb{Q}$ be a finite Galois extension, with ring of integers $\mathcal{O}_{L}$ and Galois $\operatorname{group} \Gamma:=\operatorname{Gal}(L / \mathbb{Q})$.

Definition $2.1([9], \S 2)$. We define $A(L)$ to be the set of functions $g: \Gamma \rightarrow L$ satisfying

$$
g\left(\sigma \tau \sigma^{-1}\right)=\sigma(g(\tau))
$$

for all $\sigma, \tau \in \Gamma$, which is a commutative $\mathbb{Q}$-algebra under pointwise addition and multiplication.

For $g \in A(L)$, let $p$ be a rational prime unramified in $L$ that is coprime to the denominators of all values of $g$. Let $\mathfrak{P}$ be a prime of $L$ over $p$, with Frobenius element $\phi_{\mathfrak{P}} \in \Gamma$. It follows from (2.1) that the residue class

$$
g\left(\phi_{\mathfrak{P}}\right) \bmod \mathfrak{P}
$$

is fixed by $\phi_{\mathfrak{P}}$, so $(2.2)$ is an element of $\mathbb{Z} / p \mathbb{Z} \subset \mathcal{O}_{L} / \mathfrak{P}$. It can be checked that the value of $g\left(\phi_{\mathfrak{P}}\right)$ mod $\mathfrak{P}$ is independent of the choice of $\mathfrak{P} \mid p$ (see [9], $\S 4)$, and we write $g\left(\phi_{p}\right) \bmod p$ for this residue class in $\mathbb{Z} / p \mathbb{Z}$. We leave $g\left(\phi_{p}\right)$ mod $p$ undefined for the finitely many primes that are either ramified in $L$ or are not coprime to the denominators of $g$.

The following result gives equivalence of conditions (1) and (2) in the statement of Theorem 1.1.

Theorem 2.2. An element of $\mathcal{A}$ has the form $\left(a_{p} \bmod p\right)_{p}$ for some recurrent sequence $\left(a_{n}\right)$ if and only if that element of $\mathcal{A}$ can be written $\left(g\left(\phi_{p}\right)\right.$ $\bmod p$ ) for some finite Galois extension $L / \mathbb{Q}$ and some $g \in A(L)$.

Proof. $(\Longrightarrow)$ Let $\left(a_{n}\right)$ be a recurrent sequence. Then there exist column vectors $u, v$ and an invertible matrix $M$, with entries in $\mathbb{Q}$, such that

$$
a_{n}=u^{T} M^{n} v
$$

for all $n \in \mathbb{Z}$. There is a Jordan-Chevalley decomposition

$$
M=M_{s s} M_{u},
$$

where $M_{s s}$ is semi-simple, $M_{u}$ is unipotent, and $M_{s s}$ commutes with $M_{u}$. For every prime $p$ larger than the size of $M_{u}$ that is coprime to all denominators appearing in $M_{u}$, the $p$-th power $M_{u}^{p}$ is congruent to the identity matrix modulo $p$, and if in addition $p$ is coprime to denominators appearing in $u$ and $v$, then

$$
a_{p} \equiv u^{T} M_{s s}^{p} v \quad \bmod p .
$$


Let $L$ be a finite Galois extension of $\mathbb{Q}$ over which $M_{s s}$ diagonalizes, let $\lambda_{1}, \ldots, \lambda_{k} \in L$ be the eigenvalues of $M_{s s}$, and write $\Gamma=\operatorname{Gal}(L / \mathbb{Q})$. Using the Jordan normal form of $M_{s s}$, it follows from (2.3) that there are elements $b_{1}, \ldots, b_{k} \in L$ such that

$$
a_{p} \equiv \sum_{i} b_{i} \lambda_{i}^{p} \bmod p
$$

and $\Gamma$ permutes the pairs $b_{i}, \lambda_{i}$, i.e. the element

$$
\alpha:=\sum_{i} b_{i} \otimes \lambda_{i} \in L \otimes_{\mathbb{Q}} L
$$

is invariant under the diagonal action of $\Gamma$. There is a canonical isomorphism

$$
\begin{aligned}
\varphi: L \otimes_{\mathbb{Q}} L & \rightarrow \operatorname{Hom}(\Gamma, L), \\
x \otimes y & \mapsto(\sigma \mapsto x \sigma(y)),
\end{aligned}
$$

taking the $\Gamma$-invariant elements of $L \otimes L$ to $A(L)$, and we let $g=\varphi(\alpha) \in$ $A(L)$. If $p$ is a rational prime unramified in $L$ coprime to every denominator of the values of $g$, then for every prime $\mathfrak{P}$ of $L$ over $p$,

$$
\begin{aligned}
g\left(\phi_{\mathfrak{P}}\right) & =\sum_{i} b_{i} \phi_{\mathfrak{P}}\left(\lambda_{i}\right) \\
& \equiv \sum_{i} b_{i} \lambda_{i}^{p} \quad \bmod \mathfrak{P} \\
& \equiv a_{p} \quad \bmod \mathfrak{P} .
\end{aligned}
$$

Thuse we have $\left(a_{p} \bmod p\right)=\left(g\left(\phi_{p}\right) \bmod p\right)$.

$(\Longleftarrow)$ Suppose $g \in A(L)$ is given, and let

$$
\varphi^{-1}(g)=\sum b_{i} \otimes \lambda_{i} \in(L \otimes L)^{\Gamma}
$$

where we may choose $b_{i}, \lambda_{i}$ such that the pairs $\left(b_{i}, \lambda_{i}\right)$ are permuted by $\Gamma$. Then the sequence

$$
a_{n}:=\sum_{i} b_{i} \lambda_{i}^{n}
$$

is recurrent, and takes values in $\mathbb{Q}$ because the pairs $\left(b_{i}, \lambda_{i}\right)$ are permuted by $\Gamma$. By the computation above, we see that

$$
a_{p} \equiv g\left(\phi_{p}\right) \quad \bmod p
$$

for all sufficiently large $p$. This completes the proof.

Remark 2.3. Let $L / \mathbb{Q}$ be a finite Galois extension. In the language of motives, the ring $A(L)$ defined in $\S 2$ is the ring of de Rham motivic periods of Spec $L$ (see [2], §1.2, and [9], §5). There is a ring homomorphism

$$
\begin{aligned}
\operatorname{per}_{\mathcal{A}}: A(L) & \rightarrow \mathcal{A}, \\
g & \mapsto\left(g\left(\phi_{p}\right) \quad \bmod p\right)_{p},
\end{aligned}
$$

which is an example of an $\mathcal{A}$-valued period map (see [10], §5). 


\section{Proofs OF THE THEOREMS}

In this section we prove Corollary 1.3, and Theorems 1.4 and 1.6.

Proof of Corollary 1.3. Suppose $\left(a_{n}\right)$ is a recurrent sequence. Let $L / \mathbb{Q}$ and $g \in A(L)$ be as in the statement of Theorem 2.2. Then for all primes $p$ unramified in $L$ coprime to the numerators and denominators of all nonzero values of $L$ and all $\mathfrak{P} \mid p$, we have

$$
a_{p} \equiv 0 \bmod p \Leftrightarrow g\left(\phi_{\mathfrak{P}}\right)=0 .
$$

So we may take $C=\{\sigma \in \operatorname{Gal}(L / \mathbb{Q}): g(\sigma)=0\}$, which is a union of conjugacy classes by (2.1).

Conversely, suppose $L / \mathbb{Q}$ and $C \subset \operatorname{Gal}(L / \mathbb{Q})$ are given. Let $g \in A(L)$ be the characteristic function of $C$, and let $a_{n}$ be a recurrent sequence such that

$$
a_{p} \equiv g\left(\phi_{p}\right) \quad \bmod p
$$

for all but finitely many $p$ (which exists by Theorem 2.2). Then $\left\{p: a_{p} \equiv 0\right.$ mod $p\}$ coincides with $\left\{p: \phi_{p} \subset C\right\}$ up to a finite set. We can multiply the sequence $\left(a_{n}\right)$ through by a constant rational number to modify $\left\{p: a_{p} \equiv 0\right.$ $\bmod p\}$ by any finite set. This completes the proof.

Proof of Theorem 1.4. Suppose $\left(a_{p}\right)_{p} \in \mathcal{P}_{\mathcal{A}}^{0}$ is given. By Theorem 2.2, we can find $L / \mathbb{Q}$ and $g \in A(L)$ such that $a_{p} \equiv g\left(\phi_{p}\right) \bmod p$ for all sufficiently large $p$. Since $A(L)$ is a finite-dimensional $\mathbb{Q}$-algebra, there is a non-zero $f(x) \in \mathbb{Q}[x]$ such that $f(g)=0$, which implies

$$
f\left(a_{p}\right) \equiv f\left(g\left(\phi_{p}\right)\right) \equiv 0 \quad \bmod p
$$

for all sufficiently large $p$. We can scale $f(x)$ by a rational constant to make (3.1) hold for all $p$.

Now suppose we are given $f(x) \in \mathbb{Q}[x]$ with $f\left(a_{p}\right) \equiv 0 \bmod p$ for all $p$. There are infinitely many primes $p$ that split completely in $L$, and for all but finitely many of these $p$, we have

$$
f\left(a_{p}\right) \equiv f(g(1)) \equiv 0 \quad \bmod p,
$$

where $1 \in \operatorname{Gal}(L / \mathbb{Q})$ is the identity element. Since (3.2) holds for arbitrarily large $p$, it follows that $f(g(1))=0$. Finally, $(2.1)$ implies that $g(1) \in \mathbb{Q}$, so we conclude $f(x)$ has a rational root.

Before proving Theorem 1.6, we need some preliminary results. Suppose $f(x) \in \mathbb{Q}[x]$ is monic, let $L / \mathbb{Q}$ be a finite Galois extension over which $f(x)$ splits into linear factors, and define $\Gamma:=\operatorname{Gal}(L / \mathbb{Q})$. Let $\alpha_{1}, \ldots, \alpha_{n}$ be the roots of $f$ in $L$, and for $1 \leq i \leq n$ set $\Gamma_{i}=\operatorname{Gal}\left(L / \mathbb{Q}\left(\alpha_{i}\right)\right) \subset \Gamma$.

Lemma 3.1. Let $p$ be a rational prime unramified in $L$ that is coprime to the denominators of coefficients of $f$. Then $f(x)$ has a root modulo $p$ if and 
only if the Frobenius conjugacy class $\phi_{p} \subset \Gamma$ is contained in

$$
S_{1}:=\bigcup_{i} \Gamma_{i}
$$

Proof. There is a root of $f(x)$ in $\mathbb{Z} / p \mathbb{Z}$ if and only if for some (equivalently, every) prime $\mathfrak{P}$ of $L$ over $p$, there is some $i$ for which $\alpha_{i} \bmod \mathfrak{P}$ is in $\mathbb{Z} / p \mathbb{Z} \subset \mathcal{O}_{L} / \mathfrak{P}$. Now, $\alpha_{i} \bmod \mathfrak{P}$ is in $\mathbb{Z} / p \mathbb{Z}$ if and only if $\phi_{\mathfrak{P}}\left(\alpha_{i}\right)=\alpha_{i}$, which happens if and only if $\phi_{\mathfrak{P}} \in \Gamma_{i}$. So $f$ has a root in $\mathbb{Z} / p \mathbb{Z}$ if and only if there exists $\mathfrak{P} \mid p$ with $\phi_{\mathfrak{P}} \in \bigcup \Gamma_{i}$. Since $\bigcup \Gamma_{i}$ is closed under conjugation, this is equivalent to the condition that $\phi_{p} \subset \bigcup \Gamma_{i}$.

We also need the following fact.

Lemma 3.2. Define a set

$$
S_{2}:=\bigcup_{i}\left\{\sigma \in \Gamma: C_{\Gamma}(\sigma) \subset \Gamma_{i}\right\},
$$

where $C_{\Gamma}(\sigma)$ is the centralizer of $\sigma$ inside $\Gamma$. The for every $g \in A(L)$, we have

$$
\{\sigma \in \Gamma: f(g(\sigma))=0\} \subseteq S_{2},
$$

and there exists $g \in A(L)$ for which (3.3) is an equality of sets.

Proof. If $f(g(\sigma))=0$, then $g(\sigma)=\alpha_{i}$ for some $i$. By (2.1), $g(\sigma)$ is fixed by $C_{\Gamma}(\sigma)$, so we must have $C_{\Gamma}(\sigma) \subset \operatorname{Gal}\left(L / \mathbb{Q}\left(\alpha_{i}\right)\right)=H_{i}$. This proves the containment (3.3). To show that we can choose $g \in A(L)$ for which (3.3) is equality, let $\sigma_{1}, \ldots, \sigma_{k} \in \Gamma$ be a system of conjugacy class representatives. For $1 \leq j \leq k$, if there does not exist $i$ for which $C_{\Gamma}\left(\sigma_{j}\right) \subset H_{i}$, then define $g$ to be 0 on the conjugacy class of $\sigma_{j}$. If there does exist $i$, the define $g$ on the conjugacy class of $\sigma_{j}$ by

$$
g\left(\tau \sigma_{j} \tau^{-1}\right)=\tau\left(\alpha_{i}\right)
$$

We have $g \in A(L)$ and $\{\sigma: f(g(\sigma))=0\}=S_{2}$.

We also need a group-theoretic fact about wreath products.

Lemma 3.3. Let $\Gamma$ and $A$ be finite groups, with $A$ abelian, and consider the wreath product

$$
\Gamma^{\prime}:=A^{\Gamma} \rtimes \Gamma .
$$

Let $\pi: \Gamma^{\prime} \rightarrow \Gamma$ be the projection. Then at least

$$
\left(1-\frac{|\Gamma|^{2}}{|A|}\right)\left|\Gamma^{\prime}\right|
$$

elements $\xi \in \Gamma^{\prime}$ satisfy

$$
\pi\left(C_{\Gamma^{\prime}}(\xi)\right) \subset\langle\pi(\xi)\rangle
$$


Proof. We identify elements of $\Gamma^{\prime}$ with pairs $(\varphi, \sigma)$, where $\varphi: \Gamma \rightarrow A$ and $\sigma \in \Gamma$. Under this identification, multiplication in $\Gamma^{\prime}$ is given by

$$
(\varphi, \sigma) \circ(\psi, \tau)=\left(\varphi+\psi \circ R_{\sigma}, \sigma \tau\right)
$$

(here $R_{\sigma}: \Gamma \rightarrow \Gamma$ is right multiplication by $\sigma$ ). A direct computation shows that $(\varphi, \sigma),(\psi, \tau) \in \Gamma^{\prime}$ commute if and only if $\sigma$ and $\tau$ commute and

$$
\varphi-\varphi \circ R_{\tau}=\psi-\psi \circ R_{\sigma} .
$$

For $\eta: \Gamma \rightarrow A$, there exists $\psi: \Gamma \rightarrow A$ with $\eta=\psi-\psi \circ R_{\sigma}$ if and only if

$$
\sum_{n=0}^{\operatorname{ord}(\sigma)-1} \eta \circ R_{\sigma^{n}}=0 .
$$

Combining (3.5) and (3.6), we see that, if $\varphi, \sigma$, and $\tau$ are fixed, then there exists $\psi$ such that $(\varphi, g)$ and $(\psi, h)$ commute if and only if

$$
\sum_{n=0}^{\operatorname{ord}(\sigma)-1}\left(\varphi \circ R_{\sigma^{n} \tau}-\varphi \circ R_{\sigma^{n}}\right)=0 .
$$

For each $\sigma, \tau \in \Gamma$, define a group homomorphism

$$
\begin{aligned}
\chi_{\sigma, \tau}: A^{\Gamma} & \rightarrow A, \\
\varphi & \mapsto \sum_{n=0}^{\operatorname{ord}(\sigma)-1}\left(\varphi\left(\sigma^{n} \tau\right)-\varphi\left(\sigma^{n}\right)\right) .
\end{aligned}
$$

If $\tau \notin\langle\sigma\rangle$, then the elements $\sigma^{n}$ and $\sigma^{n} \tau$ are all distinct. In this case $\chi_{\sigma, \tau}$ is seen to be surjective, and the kernel of $\chi_{\sigma, \tau}$ has index $|A|$ in $A^{\Gamma}$. It follows from (3.7) that, for fixed $\tau, \sigma$ with $\tau \notin\langle\sigma\rangle$, there are at most $|A|^{|\Gamma|-1}$ functions $\varphi: \Gamma \rightarrow A$ for which $\tau \in \pi\left(C_{\Gamma^{\prime}}((\sigma, \varphi))\right.$. Taking the union over all $\sigma, \tau \in \Gamma$ with $\tau \notin\langle\sigma\rangle$, we find that the number of elements $\xi \in \Gamma^{\prime}$ for which (3.4) does not hold is at most

$$
|\Gamma|^{2}|A|^{|\Gamma|-1}
$$

This completes the proof.

We are now ready to prove Theorem 1.6.

Proof of Theorem 1.6. Suppose $f(x) \in \mathbb{Q}[x]$. It is obvious that

$$
\delta\left(\left\{p: f\left(a_{p}\right) \equiv 0 \bmod p\right\}\right) \leq \delta(\{p: f \text { has a root } \bmod p\})
$$

for all $\left(a_{p}\right)_{p} \in \mathcal{P}_{\mathcal{A}}^{0}$. We need to show that the inequality (3.8) is strict if $f(x)$ has no rational roots, and that we can choose $\left(a_{p}\right)_{p} \in \mathcal{P}_{\mathcal{A}}^{0}$ to make (3.8) arbitrarily close to an equality. 
Suppose $f(x)$ has no rational roots. For $\left(a_{p}\right)_{p} \in \mathcal{P}_{\mathcal{A}}^{0}$, let $L / \mathbb{Q}$ and $g \in A(L)$ be such that $a_{p} \equiv g\left(\phi_{p}\right) \bmod p$, and let $\Gamma \supset S_{1} \supset S_{2}$ be as in the statements of Lemmas 3.1 and 3.2. By the Chebotarev density theorem,

$$
\begin{gathered}
\delta(\{p: f \text { has a root modulo } p\})=\frac{\# S_{1}}{\# \Gamma}, \\
\max _{g \in A(L)} \delta\left(\left\{p: f\left(g\left(\phi_{p}\right)\right) \equiv 0 \bmod p\right\}\right)=\frac{\# S_{2}}{\# \Gamma} .
\end{gathered}
$$

We get strictness of (3.8) because the identity element of $\Gamma$ is in $S_{1}$ but not in $S_{2}$.

To show that (3.8) is sharp, we pass from $L$ to an extension $L^{\prime} / L$ with the property that in $\operatorname{Gal}\left(L^{\prime} / \mathbb{Q}\right)$, most elements have small centralizers (in a sense to be made precise). For $L^{\prime} / \mathbb{Q}$ a finite Galois extension containing $L$, write $\Gamma^{\prime}=\operatorname{Gal}\left(L^{\prime} / \mathbb{Q}\right)$ and $\pi: \Gamma^{\prime} \rightarrow \Gamma$ for the restriction map. Let

$$
\Gamma_{i}^{\prime}=\pi^{-1}\left(\Gamma_{i}\right)=\operatorname{Gal}\left(L^{\prime} / \mathbb{Q}\left(\alpha_{i}\right)\right) \subset \Gamma^{\prime},
$$

for $i=1, \ldots, n$. If an element $\sigma \in \Gamma_{i}^{\prime}$ satisfies

$$
\pi\left(C_{\Gamma^{\prime}}(\sigma)\right) \subset\langle\pi(\sigma)\rangle
$$

then $C_{\Gamma^{\prime}}(\sigma) \subset \Gamma_{i}^{\prime}$. We will show that for every $\epsilon>0$, we can choose the $L^{\prime} / L$ so that $(3.9)$ holds for at least $(1-\epsilon)\left|\Gamma^{\prime}\right|$ elements $\sigma$ of $\Gamma^{\prime}$. This will prove the theorem.

Let $\epsilon>0$ be given, and choose a positive integer $r$ such that

$$
\frac{|\Gamma|^{2}}{2^{r}}<\epsilon
$$

Let $p_{1}, \ldots, p_{r}$ be distinct rational primes that split completely in the Hilbert class field of $L$. For each $i$, let $\beta_{i} \in \mathcal{O}_{L}$ be a generator for a (necessarily degree 1 and principal) prime of $L$ over $p_{i}$. Let $L^{\prime}$ be the extension of $L$ obtained by adjoining a square root of $\sigma\left(\beta_{i}\right)$ for all $\sigma \in \Gamma$ and $1 \leq i \leq r$. Then $\Gamma^{\prime}$ is isomorphic to a wreath product

$$
\Gamma^{\prime} \cong A^{\Gamma} \rtimes \Gamma,
$$

with $A=(\mathbb{Z} / 2)^{r}$. The result now follows from Lemma 3.3.

\section{PERIODS}

In this section we prove the equivalence of conditions (1) and (3) of Theorem 1.1. We also explain why $\mathcal{P}_{\mathcal{A}}^{0}$ is analogous to $\overline{\mathbb{Q}}$, and we explain how to obtain other analogues of periods inside $\mathcal{A}$.

4.1. Dimension 0. Let $L / \mathbb{Q}$ be a finite Galois extension, with ring of integers $\mathcal{O}_{L}$. There is an isomorphism of $\mathcal{A}$-algebras

$$
L \otimes_{\mathbb{Q}} \mathcal{A} \cong \frac{\prod_{p} \mathcal{O}_{L} / p \mathcal{O}_{L}}{\bigoplus_{p} \mathcal{O}_{L} / p \mathcal{O}_{L}} .
$$

For each rational prime $p$, the $p$-th power map is a $\mathbb{Z} / p \mathbb{Z}$-algebra endomorphism $F_{p, L}$ of $\mathcal{O}_{L} / p \mathcal{O}_{L}$, which is an automorphism if $p$ is unramified in $L$. 
Definition 4.1. The $\mathcal{A}$-valued Frobenius automorphism is the $\mathcal{A}$-algebra automorphism $F_{\mathcal{A}, L}$ of $L \otimes_{\mathbb{Q}} \mathcal{A}$ induced by $F_{p, L}$ in the $p$-th factor.

If we choose a basis for $L$ as a $\mathbb{Q}$-vector space, we can represent $F_{\mathcal{A}, L}$ by a square matrix with entries in $\mathcal{A}$, and the $\mathbb{Q}$-span of the matrix entries does not depend on the choice of basis.

Theorem 4.2. For each finite Galois extension $L / \mathbb{Q}$, the $\mathbb{Q}$-span of the matrix entries for $F_{\mathcal{A}, L}$ is equal to the set of elements $\left(g\left(\phi_{p}\right) \bmod p\right)_{p} \in \mathcal{A}$ for $g \in A(L)$.

Proof. The $\mathbb{Q}$-span of matrix coefficients for $F_{\mathcal{A}, L}$ is the image of the map

$$
\begin{aligned}
L^{\vee} \otimes_{\mathbb{Q}} L & \rightarrow \mathcal{A}, \\
\varphi \otimes y & \mapsto\left(\varphi\left(y^{p}\right) \bmod p\right)_{p} .
\end{aligned}
$$

Here $L^{\vee}$ is the $\mathbb{Q}$-linear dual of $L$. The trace form induces an isomorphism of $L$ with $L^{\vee}$, so the image of (4.1) is equal to the image of

$$
\begin{aligned}
& L \otimes L \rightarrow \mathcal{A} \\
& x \otimes y \mapsto\left(\left(\sum_{\sigma \in \Gamma} \sigma\left(x y^{p}\right)\right) \bmod p\right)_{p},
\end{aligned}
$$

where $\Gamma=\operatorname{Gal}(L / \mathbb{Q})$.

It follows from the proof of Theorem 2.2 that $\left\{\left(g\left(\phi_{p}\right) \bmod p\right)_{p}\right\}$ is equal to the image of the map

$$
\begin{aligned}
(L \otimes L)^{\Gamma} & \rightarrow \mathcal{A} \\
\sum_{i} x_{i} \otimes y_{i} & \mapsto\left(\left(\sum_{i} x_{i} y_{i}^{p}\right) \bmod \mathfrak{P}\right)_{p},
\end{aligned}
$$

where for each $p$ we have chosen a prime $\mathfrak{P}$ of $L$ over $p$. The result now follows from the fact that

$$
\begin{aligned}
L \otimes L & \rightarrow(L \otimes L)^{\Gamma} \\
x \otimes y & \mapsto \sum_{\sigma} \sigma(x) \otimes \sigma(y)
\end{aligned}
$$

is surjective.

The algebraic de Rham cohomology of $\operatorname{Spec}(L)$ (which we view as a 0 dimensional algebraic variety over $\mathbb{Q}$ ) is identified with $L$. Thus $\mathcal{P}_{\mathcal{A}}^{0}$ is the $\mathbb{Q}$-span of the matrix coefficients for the isomorphism

$$
H_{d R}^{0}(\operatorname{Spec}(L)) \otimes \mathcal{A} \stackrel{\sim}{\rightarrow} H_{d R}^{0}(\operatorname{Spec}(L)) \otimes \mathcal{A},
$$

for $L$ ranging over the finite Galois extensions of $\mathbb{Q}$. If instead we look at de Rham-Betti comparison isomorphism

$$
H_{d R}^{0}(\operatorname{Spec}(L)) \otimes \mathbb{C} \stackrel{\sim}{\rightarrow} H_{B}^{0}(\operatorname{Spec}(L)) \otimes \mathbb{C}
$$


for varying $L$, the $\mathbb{Q}$-span of the matrix coefficients is $\overline{\mathbb{Q}}$. For this reason $\mathcal{P}_{\mathcal{A}}^{0} \subset \mathcal{A}$ is analogous to $\overline{\mathbb{Q}} \subset \mathbb{C}$. By contrast, the integral closure of $\mathbb{Q}$ inside $\mathcal{A}$ is uncountable.

4.2. Positive dimension. The characterization of $\mathcal{P}_{\mathcal{A}}^{0}$ as matrix coefficients of the $\mathcal{A}$-valued Frobenius can be generalized to produce elements of $\mathcal{A}$ from varieties of positive dimension. If $X$ is a variety defined over $\mathbb{Q}$ and $i \geq 0$ is an integer, the algebraic de Rham cohomology $H_{d R}^{i}(X)$ is finite-dimensional vector space over $\mathbb{Q}$, and for all sufficiently large $p$ there is a distinguished automorphism

$$
F_{p, X}: H_{d R}^{i}(X) \otimes \mathbb{Q}_{p} \stackrel{\sim}{\rightarrow} H_{d R}^{i}(X) \otimes \mathbb{Q}_{p}
$$

coming from crystalline cohomology (see [6]). Matrix coefficients for $F_{p, X}$ with respect to a $\mathbb{Q}$-basis are (one type of) $p$-adic periods of $X$. Each matrix coefficient for $F_{p, X}$ is $p$-integral for all sufficiently large $p$, so reduction modulo $p$ (for all large $p$ at once) gives an element of $\mathcal{A}$. These elements are called $\mathcal{A}$-valued periods in [10]. It is convenient to assemble the maps $F_{p, X}$ to form an $\mathcal{A}$-valued Frobenius map

$$
F_{\mathcal{A}, X}: H_{d R}^{i}(X) \otimes \mathcal{A} \rightarrow H_{d R}^{i}(X) \otimes \mathcal{A},
$$

whose matrix coefficients are $\mathcal{A}$-valued periods (the map $F_{\mathcal{A}, X}$ is no longer an isomorphism). Details can be found in [10], $\S 6$.

If we instead use the de Rham-Betti comparison isomorphism

$$
\operatorname{comp}_{X}: H_{d R}^{i}(X) \otimes \mathbb{C} \stackrel{\sim}{\rightarrow} H_{B}^{i}(X) \otimes \mathbb{C},
$$

matrix coefficients are the ordinary (complex) periods of $X$. So in this analogy $\mathcal{A}$ corresponds to $\mathbb{C}$, and $F_{\mathcal{A}, X}$ corresponds to comp $p_{X}$. Define $\mathcal{P}_{\mathcal{A}} \subset$ $\mathcal{A}$ (resp. $\mathcal{P}_{\mathbb{C}} \subset \mathbb{C}$ ) to be the $\mathbb{Q}$-span of the matrix coefficients for $F_{\mathcal{A}, X}$ (resp. $\operatorname{comp}_{X}$ ), as $X$ ranges through all varieties over $\mathbb{Q}$. By taking $X$ to have dimension 0 we see that $\mathcal{P}_{\mathcal{A}}^{0} \subset \mathcal{P}_{\mathcal{A}}$ and $\overline{\mathbb{Q}} \subset \mathcal{P}_{\mathbb{C}}$.

The period conjecture of Grothendieck (see [1], §7.5) would imply that there is a $\mathbb{Q}$-algebra homomorphism

$$
\Delta: \mathcal{P}_{\mathbb{C}} \rightarrow \mathcal{P}_{\mathbb{C}} \otimes_{\mathbb{Q}} \mathcal{P}_{\mathcal{A}}
$$

Concretely, fix a variety $X$ and bases for $H_{d R}^{i}(X)$ and $H_{d R}^{i}(X)$, say of length $n$. Write $F_{\mathcal{A}, X}$ and $\operatorname{comp}_{X}$ as matrices $\left(\alpha_{i, j}\right) \in M_{n}(\mathcal{A})$ and $\left(\beta_{i, j}\right) \in M_{n}(\mathbb{C})$, respectively. The map $\Delta$ is then given by

$$
\Delta\left(\beta_{i, j}\right)=\sum_{k=1}^{n} \beta_{i, k} \otimes \alpha_{k, j} \in \mathcal{P}_{\mathbb{C}} \otimes_{\mathbb{Q}} \mathcal{P}_{\mathcal{A}}
$$

A priori the right hand side of (4.2) might depend on $X, i$, and $j$, but the the period conjecture implies that in fact the right hand side depends only on the value $\beta_{i, j} \in \mathcal{P}_{\mathbb{C}}$.

Every algebraic number occurs as a matrix coefficient for comp $p_{X}$ for some 0-dimensional $X$. Since the $\mathcal{A}$-valued periods of this $X$ are in $\mathcal{P}_{\mathcal{A}}^{0}$, this implies 
$\Delta$ takes $\overline{\mathbb{Q}} \subset \mathcal{P}_{\mathbb{C}}$ into $\mathcal{P}_{\mathcal{A}}^{0} \otimes_{\mathbb{Q}} \mathcal{P}_{\mathbb{C}}$. So the truth of the period conjecture would imply that if we see an algebraic number as a complex period of an arbitrary variety, we will also see elements of $\mathcal{P}_{\mathcal{A}}^{0}$ in the $\mathcal{A}$-valued periods of that variety.

\section{ACKNOWLEDGEMENTS}

We thank Jeffrey Lagarias for helpful comments. We thank the anonymous referee for helpful suggestions on the structure of the paper.

\section{REFERENCES}

[1] Yves André. Une introduction aux motifs: motifs purs, motifs mixtes, périodes. 2004.

[2] Francis Brown. Single-valued motivic periods and multiple zeta values. In Forum of Mathematics, Sigma, volume 2. Cambridge University Press, 2014.

[3] David Jarossay. An explicit theory of $\pi_{1}^{\text {un,crys }}\left(\mathbb{P}^{1}-\left\{0, \mu_{N}, \infty\right\}\right)-$ II-3 : Sequences of multiple harmonic sums viewed as periods, 2016, arXiv:1601.01159.

[4] Masanobu Kaneko. Finite multiple zeta values (in Japanese). RIMS Kkyroku Bessatsu B68 (2017) 175-190.

[5] Masanobu Kaneko and Don Zagier. Finite multiple zeta values. in preparation.

[6] Kiran S Kedlaya. p-adic cohomology. Algebraic Geometry, Seattle 2005: 2005 Summer Research Institute, July 25-August 12, 2005, University of Washington, Seattle, Washington, 2:667, 2009.

[7] Maxim Kontsevich. Holonomic $\mathcal{D}$-modules and positive characteristic. Japanese Journal of Mathematics, 4(1):1-25, 2009.

[8] Maxim Kontsevich and Don Zagier. Periods. In Mathematics unlimited2001 and beyond, pages 771-808. Springer, 2001.

[9] Julian Rosen. A choice-free absolute Galois group and Artin motives. 2017, arXiv:1706.06573.

[10] Julian Rosen. Sequential periods of the crystalline Frobenius. 2018, arXiv:1805.01885.

[11] Kenji Sakugawa. On modified finite polylogarithms. J. Number Theory, 201:190-205, 2019.

[12] Jean-Pierre Serre. Lectures on $N_{X}(p)$. AK Peters/CRC Press, 2016.

[13] Jianqiang Zhao. Multiple zeta functions, multiple polylogarithms and their special values, volume 12. World Scientific, 2016.

E-mail address: julianrosen@gmail.com 